\title{
Simulação do impacto da mudança climática sobre a água disponível do solo em agroecossistemas de trigo, soja e milho em Santa Maria, RS
}

\author{
Simulation of the impact of the climate change on the fraction of transpirable soil water in wheat, \\ soybean, and maize agroecosystems at Santa Maria, RS, Brazil
}

\author{
Nereu Augusto Streck ${ }^{1}$ Cleber Maus Alberto
}

\section{RESUMO}

O impacto de uma possível mudança climática sobre a água disponível em agroecossistemas é um assunto de interesse no planejamento do agronegócio. O objetivo deste estudo foi simular o impacto da mudança climática sobre o balanço de água no solo em agroecossistemas de trigo, soja e milho em Santa Maria, RS. Foram criados cenários meteorológicos dobrando-se a quantidade de $\mathrm{CO}_{2}$ com diferentes aumentos de temperatura do ar com e sem aumento de precipitação pluvial. A água disponível no solo foi representada pela fração de água transpirável no solo pelas plantas (FATS) e calculada com modelos matemáticos disponíveis na literatura. Os resultados mostraram que o aumento de temperatura diminui a FATS e esta diminuição é mais preocupante nas culturas de verão (soja e milho) do que na cultura do trigo.

Palavras-chave: Triticum aestivum, Glycine max, Zea mays, aquecimento global, balanço hídrico do solo.

\section{ABSTRACT}

The impact of the projected climate change on soil water is an important and relevant issue for planning rural activities. The objective of this study was to simulate the fraction of transpirable soil water (FTSW) in wheat, soybean and maize agroecosystems under climate change scenarios in Santa Maria, RS. Air temperature and rainfall were modified to generate climate change scenarios. FTSW was simulated with models available in the literature. The results showed that an increase in air temperature decreases FTSW and this decrease is more evident in soybean and maize than in wheat agroecosystems.

Key words: Triticum aestivum, Glycine max, Zea mays, global warming, soil water budget.

\section{INTRODUÇÃO}

A concentração de $\mathrm{CO}_{2}$ da atmosfera terrestre está aumentando, como resultado direto das atividades humanas, a uma taxa de $0,4-0,5 \%$ ano $^{-1}$ (IPCC, 1995; SOMBROEK \& GOMES, 1996), o que corresponde a um aumento de $1,0-1,8 \mu \mathrm{mol} \mathrm{CO}_{2} \mathrm{~mol}^{-1}$ ano $^{-1}$ ( IPCC, 1995; UNSWORTH \& HOGSETT, 1996). Como resultado do aumento da concentração de $\mathrm{CO}_{2}$ atmosférico, a temperatura média do ar do Planeta poderá aumentar neste período devido ao efeito estufa do $\mathrm{CO}_{2}$ atmosférico (IPCC, 1995). Estudos indicam um aumento de 1 a $6^{\circ} \mathrm{C}$ na temperatura média do ar até o final deste século em vários locais do Planeta (IPCC, 1995; MAHLMAN, 1997; IPCC, 2001), incluindo o Brasil (SIQUEIRA et al., 1994, 2000).

A possível mudança do clima terrestre pode afetar os componentes do ciclo hidrológico de ecossistemas terrestres e conseqüentemente a disponibilidade de recursos hídricos e a duração dos períodos de estiagem e enchente. O conteúdo de água no solo é um dos componentes do ciclo hidrológico que influencia diretamente a quantidade de água disponível para as culturas agrícolas, o que por sua vez afeta o seu desenvolvimento, crescimento e rendimento, e a necessidade de irrigação. No estado do Rio Grande do Sul, assim como em outros estados do Brasil, agroecossistemas são predominantemente não irrigados. Em agroecossistemas não irrigados, a disponibilidade de água para as culturas depende, entre outros fatores, da quantidade de água armazenada no solo e oriunda da precipitação pluvial. Como as culturas têm diferente eficiência de uso da água disponível no solo, é importante o estudo do balanço hídrico do solo em agroecossistemas frente a diferentes cenários de mudança climática. Esses estudos podem auxiliar no planejamento do agronegócio para lidar com este futuro desafio.

${ }^{1}$ Departamento de Fitotecnia, Centro de Ciências Rurais (CCR), Universidade Federal de Santa Maria (UFSM), 97105-900, Santa Maria, RS, Brasil. E-mail: nstreck1@smail.ufsm.br. Autor para correspondência.

${ }^{2}$ Programa de Pós-graduação em Engenharia Agrícola, CCR, UFSM, Santa Maria, RS, Brasil. 
Estudos de impacto da mudança climática são difíceis de serem realizados experimentalmente devido à limitação de equipamentos, mão-de-obra e recursos financeiros (ANDRESEN et al., 2001). Modelos matemáticos são uma simplificação da realidade que nos permitem descrever as complicadas interações que existem em agroecossistemas e, portanto, são uma ferramenta para estudos do impacto de mudanças climáticas em agroecossistemas. Existem vários modelos de simulação das culturas agrícolas, desde modelos mecanísticos de elevada complexidade e número de coeficientes (STAPPER \& HARRIS, 1989) até modelos empíricos simples (MONTEITH \& SCOTT, 1982). Em uma faixa intermediária de complexidade $\mathrm{e}$ número de coeficientes, estão os modelos mecanísticos simplificados. Os modelos de SINCLAIR (1986), AMIR \& SINCLAIR (1991) e MUCHOW \& SINCLAIR (1991), por exemplo, utilizam um reduzido número de informações, são compostos por funções matemáticas robustas e podem ser usados para diversas situações, sendo intermediários entre modelos de regressão e modelos complexos,

O trigo é a principal cultura de inverno (BERLATO \& FONTANA, 2003), enquanto a soja e o milho são as principais culturas de sequeiro de verão do estado do Rio Grande do Sul (SIQUEIRA et al., 1994). Por isso, neste trabalho, foram utilizados modelos de simulação de trigo, soja e milho. Este trabalho teve por objetivo simular o impacto da possível mudança climática sobre o balanço de água do solo em agroecossistemas de trigo, soja e milho em Santa Maria, RS, localizada na região ecoclimática da Depressão Central do estado do Rio Grande do Sul.

\section{MATERIAL E MÉTODOS}

Este estudo numérico foi realizado para as condições de Santa Maria, RS, Brasil. Utilizaram-se os dados diários de temperatura máxima e mínima, brilho solar real e precipitação pluvial coletados na Estação Climatológica Principal do $8^{\circ}$ DISME/MA localizada no campo experimental do Departamento de Fitotecnia da Universidade Federal de Santa Maria (latitude: 29\%43'S, longitude: $53^{\circ} 42^{\prime} \mathrm{W}$ e altitude: $95 \mathrm{~m}$ ) durante o período de 1969 a 2003. A densidade de fluxo de radiação solar global incidente foi estimada a partir do brilho solar real pela equação de Angstrom com coeficientes mensais para Santa Maria apresentados em ESTEFANEL et al. (1990).

Foram criados cenários climáticos para os próximos 100 anos (Tabela 1), com base em SIQUEIRA et al. (2001). O cenário sem mudança climática consistiu de um conjunto de dados sintéticos de 100 anos criados com o "weather generator" LARS-WG (SEMENOV et al., 1998) a partir dos dados observados no período de 1969 a 2003. A partir deste conjunto de dados sintéticos foram criados os cenários com aumentos de temperatura, precipitação e $\mathrm{CO}_{2}$ (Tabela 1$)$, os quais foram efetuados linearmente do início ao final dos 100 anos. Foi considerado um incremento de $\mathrm{CO}_{2}$ de 350ppm (situação atual - SIQUEIRA et al., 2000) até 700ppm (mudança climática - IPCC, 1995).

O solo considerado na simulação foi o solo da unidade de mapeamento São Pedro, um Argissolo Vermelho-Amarelo Distrófico Arênico (BRASIL, 1973), representativo da região. Os valores do conteúdo de água no solo nas diferentes camadas de solo no ponto de murcha permanente (PMP, $\mathrm{cm}^{3} \cdot \mathrm{cm}^{-3}$ ) e na capacidade de campo $\left(\mathrm{CC}, \mathrm{cm}^{3} \cdot \mathrm{cm}^{-3}\right)$, necessários nos modelos, foram retirados de NASCIMENTO (1981). O conteúdo de água disponível para as plantas foi representado pela fração percentual de água transpirável no solo pelas plantas (FATS), calculada por:

$\mathrm{FATS}=[(\mathrm{CA}-\mathrm{PMP}) /(\mathrm{CC}-\mathrm{PMP})]^{*} 100$

em que, CA é o conteúdo atual de água no solo $\left(\mathrm{cm}^{-3} \cdot \mathrm{cm}^{-3}\right)$ e os outros termos já foram definidos.

A FATS foi simulada pelos modelos de AMIR \& SINCLAIR (1991), SINCLAIR (1986) e MUCHOW \& SINCLAIR (1991) para as culturas de trigo, soja e milho, respectivamente. A profundidade máxima do sistema radicular de cada cultura foi de $1,2 \mathrm{~m}$, $0,7 \mathrm{~m}$ e $1,2 \mathrm{~m}$, para as culturas do trigo, soja e milho, respectivamente.

No modelo de AMIR \& SINCLAIR (1991), o ciclo de desenvolvimento da cultura do trigo é dividido em três sub-períodos: emergência - crescimento da haste principal, crescimento da haste principal - antese, e enchimento de grãos, com duração de 1243, 212 e $585^{\circ} \mathrm{C}$.dia, respectivamente. Entre a antese e o início do enchimento de grãos, há um intervalo de $90^{\circ} \mathrm{C}$.dia. Para o cálculo da soma térmica, em graus-dia $\left({ }^{\circ} \mathrm{C}\right.$.dia), a temperatura base $\left(\mathrm{T}_{\mathrm{b}}\right)$ foi de $0^{\circ} \mathrm{C}$ e a temperatura média diária foi calculada a partir da média aritmética da temperatura mínima e máxima diária.

O número final de folhas na haste principal e o acúmulo térmico entre o aparecimento de duas folhas sucessivas (filocrono) do trigo, necessários para o modelo de AMIR \& SINCLAIR (1991), foram de 11 folhas e $113^{\circ} \mathrm{C}$.dia, respectivamente, para a cultivar BRS 179 (BOSCO et al., 2004), genótipo de trigo recomendado para a região Sul do Brasil (EMBRAPA TRIGO, 2004a).

No modelo de simulação da cultura soja de SINCLAIR (1986), as datas de término de crescimento 
Tabela 1 - Cenários de mudança climática para Santa Maria, RS, Brasil, utilizados neste estudo numérico.

\begin{tabular}{|c|c|c|c|c|c|c|}
\hline \multirow{2}{*}{ Cenário } & \multirow{2}{*}{$\begin{array}{l}\text { Temperatura } \\
\text { média }\left({ }^{\circ} \mathrm{C}\right)\end{array}$} & \multirow{2}{*}[\mathrm{CO}_{2}]{$(\mathrm{ppm})$} & \multicolumn{4}{|c|}{ Precipitação pluvial (\%) } \\
\hline & & & Dez-Fev & Mar-Mai & Jun-Ago & Set-Nov \\
\hline 1 & - & 350 & - & - & - & - \\
\hline 2 & - & 700 & - & - & - & - \\
\hline 3 & $+2^{\circ} \mathrm{C}$ & 700 & - & - & - & - \\
\hline 4 & $+3^{\circ} \mathrm{C}$ & 700 & - & - & - & - \\
\hline 5 & $+4^{\circ} \mathrm{C}$ & 700 & - & - & - & - \\
\hline 6 & $+5^{\circ} \mathrm{C}$ & 700 & - & - & - & - \\
\hline 7 & $+6^{\circ} \mathrm{C}$ & 700 & - & - & - & - \\
\hline 8 & - & 700 & +7 & +17 & +4 & +25 \\
\hline 9 & $+2^{\circ} \mathrm{C}$ & 700 & +7 & +17 & +4 & +25 \\
\hline 10 & $+3^{\circ} \mathrm{C}$ & 700 & +7 & +17 & +4 & +25 \\
\hline 11 & $+4^{\circ} \mathrm{C}$ & 700 & +7 & +17 & +4 & +25 \\
\hline 12 & $+5^{\circ} \mathrm{C}$ & 700 & +7 & +17 & +4 & +25 \\
\hline 13 & $+6^{\circ} \mathrm{C}$ & 700 & +7 & +17 & +4 & +25 \\
\hline
\end{tabular}

foliar e início do enchimento de grãos foram simuladas com o modelo não-linear de resposta do desenvolvimento à temperatura e fotoperíodo proposto por SINCLAIR et al. (1991), utilizando-se dados da cultivar Bragg publicados em SCHNEIDER et al. (1984) para a estimativa dos coeficientes nas condições locais. No modelo de simulação da cultura do milho de MUCHOW \& SINCLAIR (1991), os coeficientes foram os mesmos da publicação original.

A simulação iniciou no dia $01 / 01$ do ano 1 e finalizou no dia $31 / 12$ do ano 100 . As datas de semeadura e os sistemas de cultivos adotados neste estudo numérico estão na tabela 2. Estas datas foram selecionadas em função da época recomendada para a cultura do trigo (EMBRAPA TRIGO, 2004a), soja (EMBRAPATRIGO, 2004b) e milho(MALUF etal., 2000) na região central do Rio Grande do Sul. Considerou-se que, durante o período de pousio, não havia vegetação em crescimento ativo sobre a superfície do solo e os restos culturais permaneceram sobre a superfície após a colheita.

O dia da colheita de cada cultivo foi considerado como sendo o dia após àquele em que o acúmulo térmico é de $233^{\circ} \mathrm{C}$.dia $\left(\mathrm{T}_{\mathrm{b}}=5^{\circ} \mathrm{C}\right)$ após a maturação fisiológica (HAMADA et al., 1999). Caso

Tabela 2 - Sistemas de cultivo e datas de semeadura das culturas assumidos neste estudo numérico.

\begin{tabular}{lcc}
\hline Cultura & Sistema de cultivo & Data de Semeadura \\
\hline Trigo & Trigo - Pousio & 14 de Junho \\
Soja & Pousio - Soja & 15 de Novembro \\
Milho & Pousio - Milho & 15 de Novembro \\
\hline
\end{tabular}

tenha ocorrido precipitação acima de $5 \mathrm{~mm}$ no dia em que os $233^{\circ} \mathrm{C}$.dia foram completados, esperaram-se dois dias após o último dia de chuva para o dia da colheita. O cálculo da decomposição dos restos culturais após a colheita foi baseado nas equações propostas por GHIDEY et al. (1985). A presença de resíduo sobre a superfície resulta na atenuação da perda de água por evaporação. A diminuição da evaporação de água do solo proporcionada pelos resíduos culturais foi calculada pela equação apresentada em GILL \& JALOTA(1996).

A resposta das culturas ao aumento de $\mathrm{CO}_{2}$ atmosférico foi considerada modificando-se a eficiência do uso de radiação (EUR) e a eficiência do uso da água (EUA). A modificação na EUA foi realizada através da redução da transpiração pelas culturas em ambiente com elevado $\mathrm{CO}_{2}$ (GOTTSCHALCK et al., 2001). No cenário sem mudança climática, utilizou-se uma EUR de $0,9 \mathrm{~g} \cdot \mathrm{MJ}^{-1}$ para o trigo (MOREIRA et al., 1999) e $1,2 \mathrm{~g} \cdot \mathrm{MJ}^{-1}$ para a soja (SINCLAIR, 1986) e o milho (MUCHOW \& SINCLAIR, 1991). Nos cenários com aumento de $\mathrm{CO}_{2}$, utilizou-se aumento de $25 \%$ na EUR para as culturas do trigo e soja e de $10 \%$ para cultura do milho (TUBIELLO et al., 2000) e a transpiração foi reduzida em $23 \%$ para o trigo (CURE \& ACOCK, 1986) e 38 e $40 \%$ para as culturas de soja e milho, respectivamente (GOTTSCHALCK et al., 2001). O aumento total na EUR e na EUA só ocorreu no final do período de simulação, quando a concentração de $\mathrm{CO}_{2}$ atingiu 700ppm, o dobro da atual. A significância da tendência da FATS em função dos anos nos diferentes cenários climáticos foi testada com o teste não paramétrico de Kendall Tau (SIEGEL, 1975). 


\section{RESULTADOS E DISCUSSÃO}

Na figura 1, são apresentados alguns dos cenários climáticos gerados e utilizados no estudo, como os cenários sem aumento de temperatura e com aumento de 3 e $6^{\circ} \mathrm{C}$ na temperatura média anual (Figura 1a) e os cenários sem e com aumento de precipitação pluviométrica acumulada anual (Figura 1b). Nota-se um aumento gradual dos dois elementos meteorológicos até atingir o aumento total no final do século. Os outros cenários de aumento de temperatura foram intermediários entre sem e com aumento de temperatura de $6^{\circ} \mathrm{C}$.
O efeito da mudança climática sobre a FATS foi variável em função do cenário e da cultura, como mostram os valores de coeficiente de correlação do teste de Kendall Tau entre FATS e anos (Tabela 3). A FATS média durante o ciclo de desenvolvimento das culturas calculada pelos modelos matemáticos geralmente apresentou tendência de diminuição com aumento de temperatura (valor negativo do coeficiente de correlação) nas três culturas (Tabela 3 ), mas apenas para o trigo no cenário de mais $6^{\circ} \mathrm{C}$ (cenário 7) e milho nos cenários de mais 5 e $6^{\circ} \mathrm{C}$ (cenário 6,7 e 13) estas tendências foram significativas $(\mathrm{P}<0,01)$. Apenas para o cenário 8 (aumento de $\mathrm{CO}_{2}$ e sem aumento de

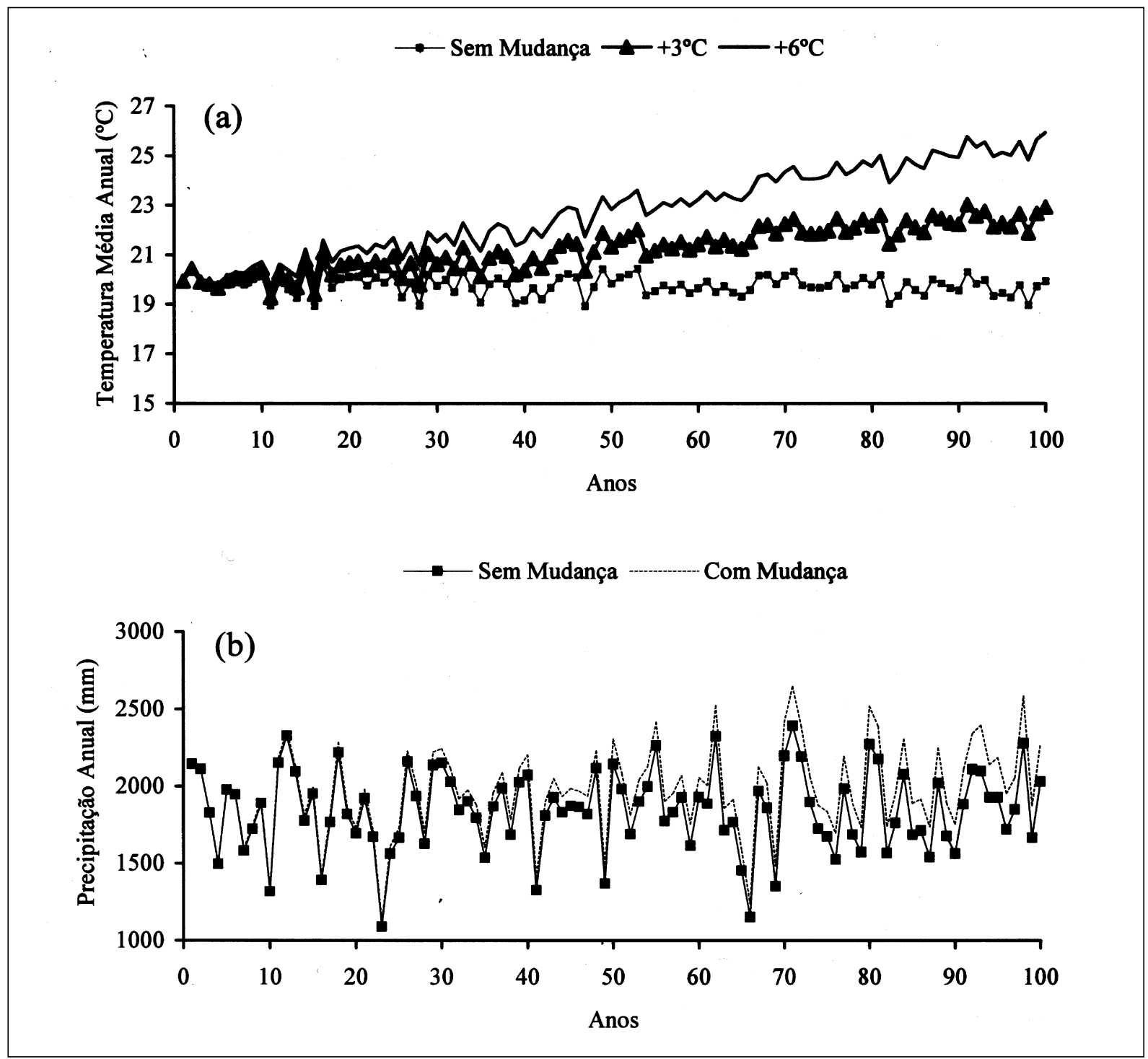

Figura 1 - Alguns cenários climáticos de temperatura média anual (a) e precipitação pluvial acumulada anual (b) para Santa Maria, RS, usados no estudo.

Ciência Rural, v.36, n.2, mar-abr, 2006. 
Tabela 3 - Valores do coeficiente de correlação de Kendall Tau entre fração percentual de água transpirável no solo (FATS) e anos em diferentes cenários de mudança climática simulada por modelos matemáticos para as culturas de trigo, soja, e milho, Santa Maria, RS. A FATS é a média durante o ciclo desenvolvimento das culturas (semeadura à colheita).

\begin{tabular}{lccc}
\hline Cenário & Trigo & Soja & Milho \\
Climático & & & \\
& & & \\
\hline 1 & $-0,051717$ & 0,013812 & $-0,001443$ \\
2 & $-0,029091$ & 0,086786 & 0,166357 \\
3 & $-0,074747$ & 0,024119 & $-0,131313$ \\
4 & $-0,096566$ & $-0,005566$ & $-0,146568$ \\
5 & $-0,116768$ & $-0,035663$ & $-0,175015$ \\
6 & $-0,149091$ & $-0,069882$ & $-0,204700^{*}$ \\
7 & $-0,179798 *$ & $-0,106164$ & $-0,236446^{*}$ \\
8 & 0,024646 & 0,130489 & $0,184910^{*}$ \\
9 & $-0,040404$ & 0,070707 & $-0,088848$ \\
10 & $-0,068687$ & 0,034426 & $-0,109050$ \\
11 & $-0,090101$ & $-0,006803$ & $-0,146155$ \\
12 & $-0,107475$ & $-0,034014$ & $-0,167182$ \\
13 & $-0,132929$ & $-0,066996$ & $-0,189858^{*}$ \\
\hline
\end{tabular}

* significativo a $1 \%$ de probabilidade de erro pelo teste $\mathrm{F}$.

temperatura) houve tendência de aumento na FATS (valor positivo do coeficiente de correlação) para todas as culturas, embora apenas para o milho este aumento foi estatisticamente significativo.

Para a cultura do trigo, a FATS foi superior a $45 \%$ em todos os cenários e na maioria dos anos superior a $60 \%$ (Figuras 2 e 3). Menores valores de FATS foram simulados para as culturas de verão, e, na maioria dos anos, os valores foram inferiores a $60 \% \mathrm{e}$ com freqüência eram entre 30 e $40 \%$ (Figuras 2 e 3). A análise dos últimos 10 anos dos cenários de mudança climática para Santa Maria (Figura 4) mostra que ocorre uma diminuição da FATS para as três culturas à medida que a temperatura do ar aumenta, mas para a cultura do trigo esta diminuição de FATS tem pequeno impacto já que, no pior cenário, a FATS é superior a 70\%. Já para as culturas de verão, a FATS no pior cenário (aumento de $6^{\circ} \mathrm{C}$ sem mudança na precipitação, Figura 4 a) é inferior a $40 \%$ para ambas as culturas. Caso o aumento de temperatura vier acompanhado de mudança na precipitação, a FATS, nas culturas de verão, aumentaria um pouco, mas ainda assim ficaria baixa e próxima de 40\% (Figura 4b). Processos fisiológicos como a transpiração, a fotossíntese e a expansão foliar começam a ser afetados devido ao fechamento estomático quando a FATS é inferior a $40 \%$ em trigo, soja e milho (SINCLAIR, 1986; AMIR \& SINCLAIR, 1991; MUCHOW \& SINCLAIR, 1991). Estes resultados indicam que a FATS não é um fator limitante para a cultura do trigo em Santa Maria, mesmo em cenários de aumento de temperatura do ar que causam a redução da FATS em níveis que ainda não afetam processos fisiológicos da planta. Isto ocorre porque, sendo uma cultura de inverno, o ciclo de desenvolvimento do trigo ocorre durante o período do ano em que, no Rio Grande do Sul, a demanda atmosférica por vapor de água é baixa em função das baixas temperaturas e baixo déficit de saturação do ar. Por outro lado, a FATS é um fator limitante para as culturas de verão (soja e milho) em Santa Maria, o que deverá se agravar caso a temperatura do ar aumente no futuro.

A maior FATS ocorreu nos cenários com aumento de $\mathrm{CO}_{2}$ mas sem aumento de temperatura do $\operatorname{ar}$ (Figura $4 \mathrm{a}$ e $4 \mathrm{~b}$ ). Isto ocorre em função do fechamento estomático em ambiente com elevado $\mathrm{CO}_{2}$, o que diminui a transpiração (UNSWORTH \& HOGSETT, 1996; STRECK, 2005). A diminuição da FATS em cenários com aumento de temperatura ocorre em função do aumento da transpiração das plantas devido ao aumento do déficit de saturação do ar entre a câmara subestomática e o ar externo à planta e pelo aumento de evaporação do solo devido ao aumento do déficit de saturação do ar acima do solo (ROSENBERG et al., 1990; STRECK, 2005),

Um aumento de temperatura de apenas $2^{\circ} \mathrm{C}$ já é suficiente para diminuir a FATS em relação ao cenário sem mudança climática para as culturas de soja e milho (Figura 4). Estes resultados indicam a fragilidade dos agroecossistemas à mudanças climáticas. Se o aumento de $\mathrm{CO}_{2}$ atmosférico não vier acompanhado por aumento de temperatura do ar, os agroecossistemas até poderão ser beneficiados, pois o aumento de $\mathrm{CO}_{2}$ aumenta a fotossíntese e economiza água no solo, o que representa maiores rendimentos para as culturas (STRECK, 2005). No entanto, se o aumento de CO vier acompanhado de aumento de temperatura, as culturas terão maior stress hídrico, mesmo que um aumento de precipitação acompanhe a mudança climática (Figura 4b). Os resultados deste estudo mostram que a possível mudança climática pode aumentar o risco, que já é alto, do agronegócio. Os efeitos negativos desta possibilidade têm maior impacto em países de economia frágil e não consolidada que compreendem a maioria dos países do bloco econômico em desenvolvimento, já que, nestes países, os subsídios agrícolas são menores e os agricultores menos capitalizados. O Brasil faz parte deste bloco econômico. 


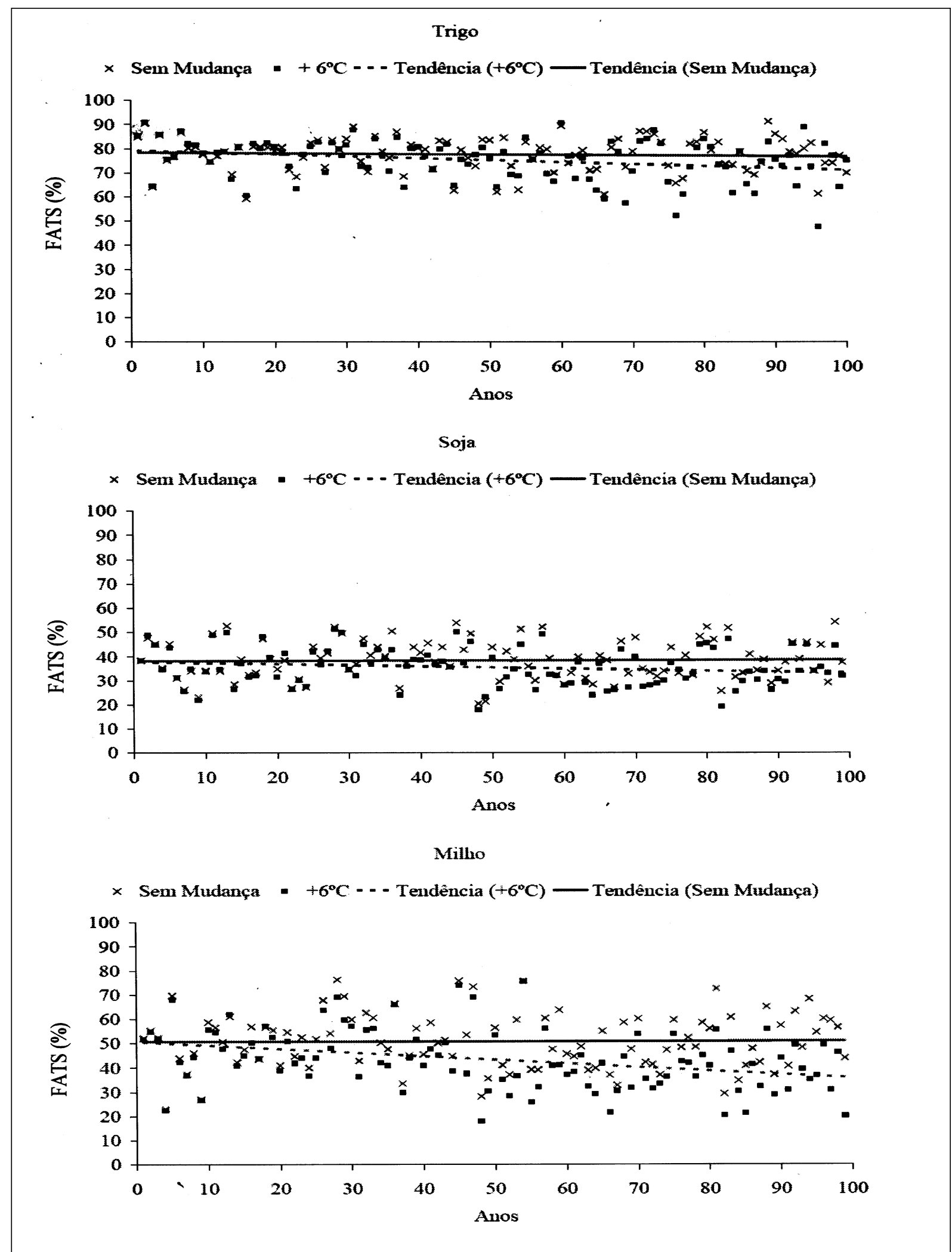

Figura 2 - Fração percentual de água transpirável no solo (FATS) simulada para as culturas de trigo, soja e milho em cenário sem mudança climática (cenário 1) e com aumento de $6^{\circ} \mathrm{C}$ na temperatura média do ar (cenário 7), Santa Maria, RS. A FATS é a média durante o ciclo de desenvolvimento das culturas (semeadura à colheita).

Ciência Rural, v.36, n.2, mar-abr, 2006. 


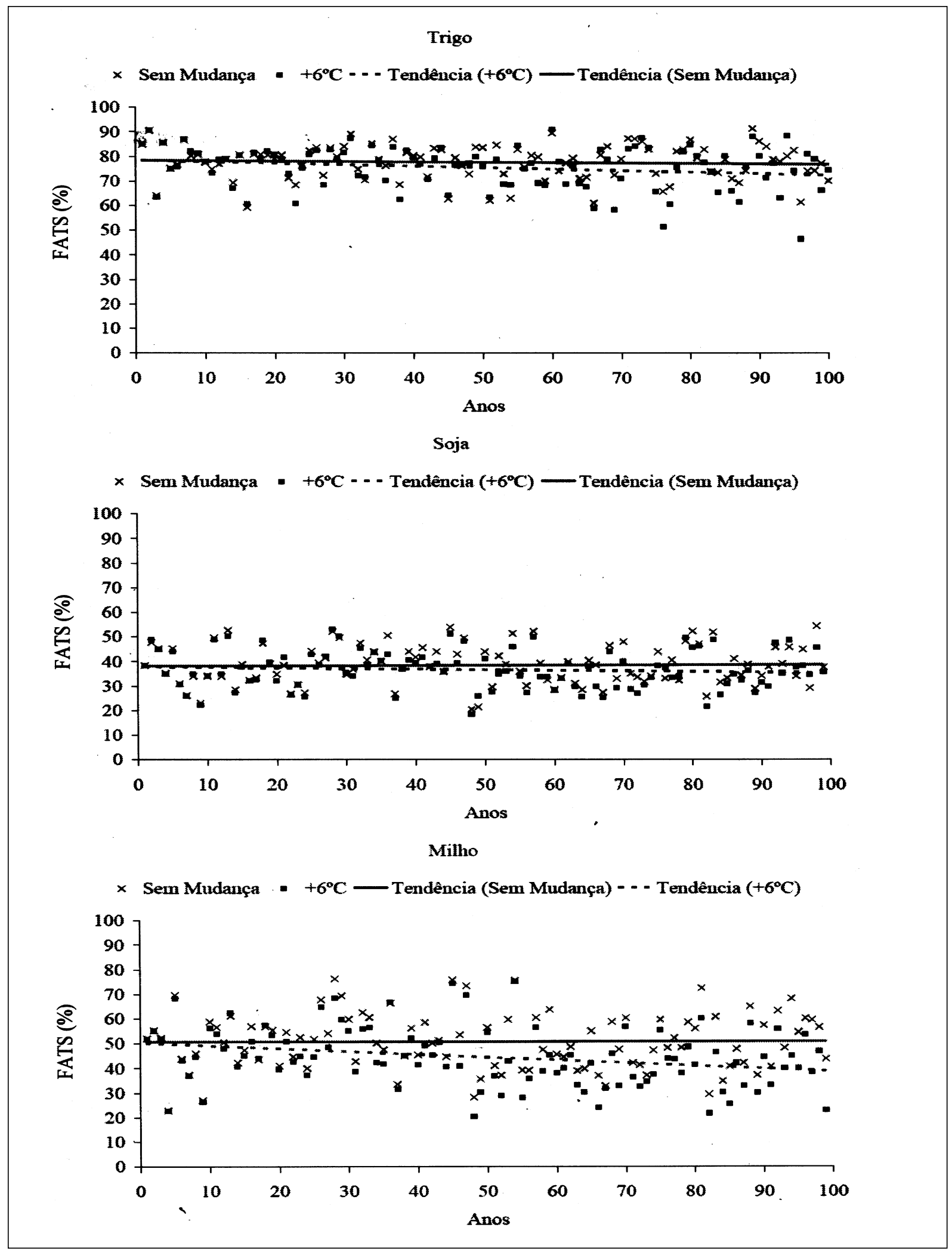

Figura 3 - Fração percentual de água transpirável no solo (FATS) simulada para as culturas do trigo, soja e milho em cenário sem mudança climática (cenário 1) e com aumento de $6^{\circ} \mathrm{C}$ na temperatura média do ar e na precipitação pluviométrica (cenário 13), Santa Maria, RS. A FATS é a média durante o ciclo de desenvolvimento das culturas (semeadura à colheita).

Ciência Rural, v.36, n.2, mar-abr, 2006. 


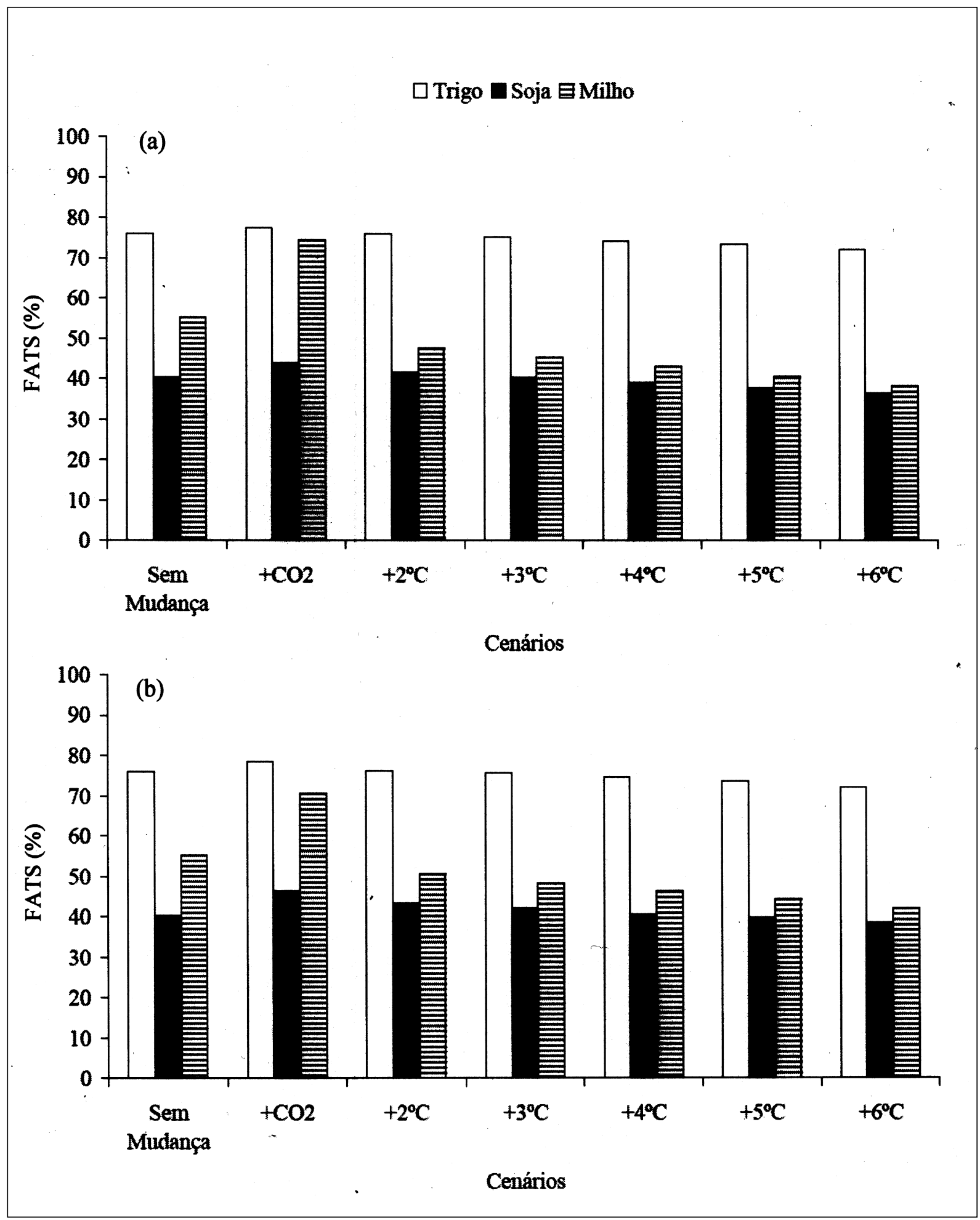

Figura 4 - Fração percentual de água transpirável no solo (FATS) simulada para as culturas de trigo, soja e milho em diferentes cenários climáticos com aumento de $\mathrm{CO}_{2}$ para Santa Maria, RS: (a) sem mudança na precipitação, (b) com mudança na precipitação. As barras representam a média dos últimos 10 anos de cada cenário. 


\section{CONCLUSÃO}

A mudança climática projetada para os próximos 100 anos em Santa Maria, RS, influenciará no balanço de água no solo em agroecossistemas de trigo, soja e milho. Aumento de $\mathrm{CO}_{2}$ associado a aumentos de temperatura de até $6^{\circ} \mathrm{C}$ tendem a diminuir o conteúdo de água no solo, e essa diminuição é mais preocupante nas culturas de verão (soja e milho) do que na cultura do trigo.

\section{AGRADECIMENTOS}

À Coordenação de Aperfeiçoamento de Pessoal de Ensino Superior (CAPES) pela concessão de bolsa ao pesquisador Alberto.

\section{REFERÊNCIAS}

AMIR, J.; SINCLAIR, T.R. A model of water limitation on spring wheat growth and yield. Field Crops Research, v.29, n.1, p.59-96, 1991.

ANDRESEN, J.A. et al. Weather Impacts on maize, soybean, and alfafa production in the Great Lakes Region, 1895-1996. Agronomy Journal, v.93, p.1059-1070, 2001.

BERLATO, M.A.; FONTANA, D.C. El Niño e La Niña: impactos no clima, na vegetação e na agricultura do Rio Grande do Sul; aplicações de previsões climáticas na agricultura. Porto Alegre: UFRGS, 2003. 110p.

BOSCO, L.C. et al. Estimativa do filocrono de algumas cultivares brasileiras de trigo. In: JORNADA ACADÊMICA INTEGRADA, 18., 2004, Santa Maria. Anais... Santa Maria: UFSM, 2004. 1 CD-ROM.

BRASIL. Levantamento de reconhecimento dos solos do estado do Rio Grande do Sul. Recife: Ministério da Agricultura/ Divisão de Pesquisa Pedológica, 1973. 431p. (Boletim técnico n.30).

CURE, J.D.; ACOCK, B. Crop response to carbon dioxide doubling: a literature survey. Agricultural and Forest Meteorology, v.38, n.1-3, p.127-145, 1986.

EMBRAPA TRIGO. Indicações técnicas da Comissão SulBrasileira de Pesquisa de Trigo - 2004. Acesso em 20 abr. 2004a. On-line. Disponível em: http://www.cnpt.embrapa.br/ resbpt04/index.htm.

EMBRAPA TRIGO. Indicações técnicas para a cultura de soja no Rio Grande do Sul e em Santa Catarina 2004/ 2005. Acesso em 20 abr. 2004b. On-line. Disponível em: http://www.cnpt.embrapa.br/i_soja.htm

ESTEFANEL, V. et al. Insolação e radiação solar em Santa Maria, RS: I - Estimativa da radiação solar global incidente a partir dos dados de insolação. Revista do Centro de Ciências Rurais, v.20, n.3-4, p.203-218, 1990.

GHIDEY, F. et al. Residue decay evaluation and prediction. TRANSACTIONS of the ASAE, v.28, n.1, p.102-105, 1985.
GILL, B.S.; JALOTA, S.K. Evaporation from soil in relation to residue rate, mixing depth, soil texture and evaporativity. Soil Technology, v.8, p.293-301, 1996.

GOTTSCHALK, J.C. et al. The simulation of canopy transpiration under doubled $\mathrm{CO}_{2}$ : The evidence and impact of feedbacks on transpiration in two 1-D soil-vegetationatmosphere-transfer models. Agriculture and Forest Meteorology, v.106, p.1-21, 2001.

HAMADA, E. et al. Avaliação do desenvolvimento do trigo em função de graus-dia. In: CONGRESSO BRASILEIRO DE AGROMETEOROLOGIA, 11., 1999, Florianópolis. Florianópolis, SC Anais... Florianópolis: Sociedade Brasileira de Agrometorologia, 1999. CD-Room.

IPCC [Intergovernmental Panel on Climate Change]. Climate change - The IPCC scientific assessment. Cambridge: Cambridge University, 1995. 288p.

IPCC [Intergovernmental Panel on Climate Change]. Climate change 2001: the scientific bases - The contribution of the working group $I$ of the third assessment report. Cambridge: Cambridge University, 2001. 944p.

MAHLMAN, J.D. Uncertainties in projections of human induced climate warnings. Science, v.278, n.5342, p.14161417, 1997.

MALUF, J.R.T. et al. Zoneamento agroclimático da cultura do milho por épocas de semeadura, no estado do Rio Grande do Sul. Pesquisa Agropecuária Gaúcha, v.6, n.1, p.39-54, 2000.

MONTEITH, J.L.; SCOTT, R.K. Wheather and yield variation of crops. In: BLAXTER, K., FOWDEN, L. Food, nutrition and climate. Englewood Cliffs: Applied science, 1982. p.127-149.

MOREIRA, A.M. et al. Eficiência do uso de radiação e índice de colheita em trigo submetido a estresse hídrico em diferentes estádios de desenvolvimento. Scientia Agrícola, v.56, n.3, p.597-603, 1999.

MUCHOW, R.C.; SINCLAIR, T.R. Water deficit effects on maize yields modeled under current and "Greenhouse" climates. Agronomy Journal, v.83, p.1052-1059, 1991.

NASCIMENTO, J.L. do. Características de umidade e disponibilidade de água do solo da unidade São Pedro, RS. 1981. 56f. Dissertação (Mestrado em Engenharia Agrícola) - Universidade Federal de Santa Maria.

ROSENBERG, N.J. et al. From climate and $\mathrm{CO}_{2}$ enrichment to evapotranspiration. In: WAGGONER, P.E. Climate change and U. S. water resources. New York: John Wiley \& Sons, 1990. p.151-175.

SCHNEIDER, F.M. et al. Comportamento de doze cultivares de soja à diferentes condições de drenagem e de irrigação subterrânea num solo hidromórfico. In: Contribuição do Centro de Ciências Rurais à XII reunião de pesquisa de soja da Região Sul. Santa Maria: UFSM/FATEC, 1984. p.29-42.

SEMENOV, M.A. et al. Comparison of the WGEN and LARSWG stochastic weather generators for diverse climates. Climate Research, v.10, n.1, p.95-107, 1998. 
SIEGEL, S. Estatística não-paramétrica. São Paulo: McGrawHill, 1975. 350p.

SINCLAIR, T.R. Water and nitrogen limitations in soybean grain production. I. Model development. Field Crops Research, v.15, n.2, p.125-141, 1986.

SINCLAIR, T.R. et al. Soybean flowering date: linear and logistic models based on temperature and photoperiod. Crop Science, v.31, p.786-790, 1991 .

SIQUEIRA, O.J.W. et al. Potential effects of global climate changes for brazilian agriculture and adaptative strategies for wheat, maize and soybean. Revista Brasileira de Agrometeorologia, v.2, n.1, p.115-129, 1994.

SIQUEIRA, O.J.W. et al. Mudanças climáticas projetadas através dos modelos GISS e reflexos na produção agricola brasileira. Revista Brasileira de Agrometeorologia, v.8, n.2, p.311-320, 2000.

SIQUEIRA, O.J.W. et al. Efeitos potenciais das mudanças climáticas na agricultura brasileira e estratégias adaptativas para algumas culturas. In: LIMA, M. A. et al. Mudanças climáticas globais e a agropecuária brasileira. EMBRAPA: Jaguariúna, SP, 2001. p.33-63.
SOMBROEK, W.G.; GOMES, R. The climate change-Agriculture conundrum. In: BAZZAZ, F.; SOMBROEK, W. Global climate change and agricultural production. New York: John Wiley \& Sons, 1996. Cap.1, p.1-14.

STAPPER, M.; HARRIS, H.C. Assessing de productivity of wheat genotypes in a Mediterranean climate, using a crop simulation model. Field Crops Research, v.20, p.129-152, 1989.

STRECK, N.A. Climate change and agroecosystems: the effect of elevated atmospheric $\mathrm{CO}_{2}$ and temperature and crop growth, development, and yield. Ciência Rural, v.35, n.3, p.730$740,2005$.

TUBIELLO, F.N. et al. Effects of climate change and elevated $\mathrm{CO}_{2}$ on cropping systems: model predictions at two Italian locations. European Journal of Agronomy, v.13, p.179189,2000 .

UNSWORTH, M.H.; HOGSETT, W.E. Combined effect of changing $\mathrm{CO}_{2}$, temperature, $\mathrm{UVB}$ radiation, and $\mathrm{O}_{3}$ on crop growth. In: BAZZAZ, F.; SOMBROEK, W. Global climate change and agricultural production. New York: John Wiley \& Sons, 1996. p.171-198. 